\title{
Splash and Bubbles for Parents App: Station Study Report
}

Tiffany Leones, Danae Kamdar, Kayla Huynh, Melissa Gedney, ${ }^{1}$ and Ximena Dominguez

June 2021

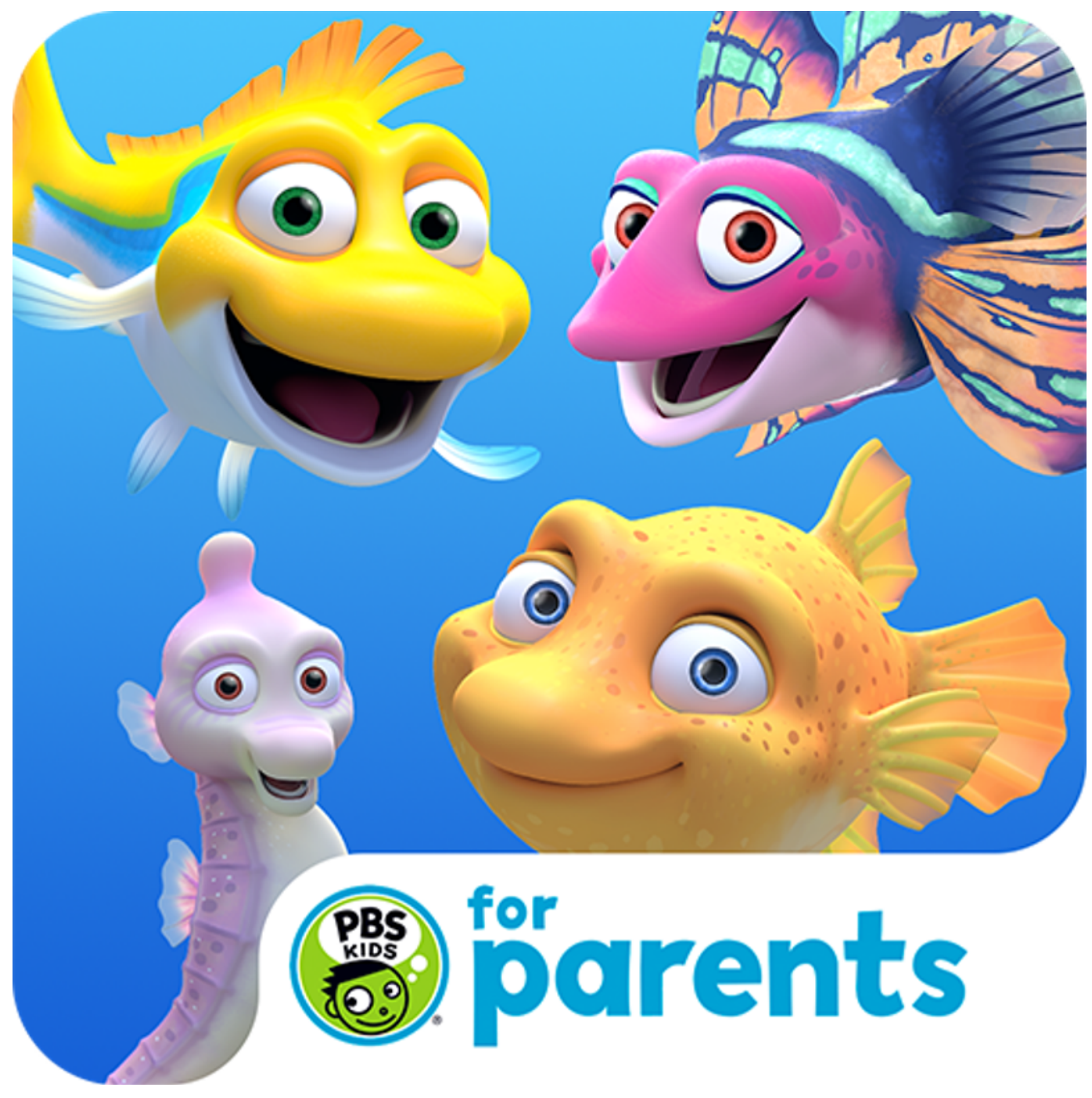

Digital

${ }^{1}$ All authors are staff of Digital Promise except for Melissa Gedney (PBS) 


\section{Suggested Citation}

Leones, T., Kamdar, D., Huynh, K., Gedney, M., \& Dominguez, X. (2021). Splash and Bubbles for Parents App: Station Study Report [Project Report]. Digital Promise. https://doi.org/10.51388/20.500.12265/120

\section{Acknowledgments}

This material is based upon work supported by the National Science

Foundation under Grant No. 1612840. Any opinions, findings, and conclusions or recommendations expressed in this material are those of the author(s) and do not necessarily reflect the views of the National Science Foundation.

\section{Contact Information}

Email: xdominguez@digitalpromise.org

Digital Promise:

Washington, DC:

1001 Connecticut Avenue NW, Suite 935

Washington, DC 20036

San Mateo, CA:

2955 Campus Dr. Suite 110

San Mateo, CA 94403

https://digitalpromise.org/ 


\section{Executive Summary}

The Splash and Bubbles for Parents app is a second-screen digital resource for parents and caregivers to support young children's learning of ocean science. This report, prepared for The Jim Henson Company, shares findings of a station study conducted to investigate the types of support parents and caregivers need when navigating and using this new digital tool. In partnership with local Public Broadcasting Service (PBS) stations, we provided parents and caregivers more detailed support around the features of the app. Overall, parents and caregivers found the app helpful for supporting their children's science learning validating the findings from the prior field study. Findings indicate that all sections of the app were used and could help promote conversations. Parents and caregivers expressed choosing to use a specific app section when they felt it was more relevant or developmentally appropriate for their child, often citing the child's interest or age as a factor. 


\section{App Introduction and Research/Development Process}

Splash and Bubbles for Parents is an app that provides resources for families to explore ocean science together. The app was designed to be used as a second-screen experience for parents and caregivers; it provides conversation starter ideas for families to use while watching the Splash and Bubbles television show. The app also includes video clips, information on ocean animals and environments, and activities designed to extend families' opportunities to learn about ocean science together. A team of media producers, early childhood learning scientists, and marine biology experts worked in collaboration with families to design the app. Throughout the design and development process, design-based research was conducted to gather information about how families engage in shared science learning experiences and how the app could support this engagement. Research activities included user studies to pilot early versions of the app and inform multiple rounds of iterative refinement.

The final stages of the research included a field study to collect evidence of promise. Findings from this study provided information about how the app supported families to talk about science together; what science concepts and practices children learned through engaging with the app and related science activities; and how families shifted their attitudes, beliefs, or practices around science and media. The field study also highlighted parents' need for support around ways to engage with and use the app given that this type of digital tool represents a new kind of resource that families may not be familiar with. To address this need, the team partnered with local Public Broadcasting Service (PBS) stations to conduct a study to investigate which types of support could be useful to families as they familiarize themselves with the app. Orientation events (described below) were implemented by stations as part of the study.

\section{Study Design}

Participating families were recruited through collaboration with local PBS stations. Families were invited to join a virtual orientation (described in detail below) and asked to complete an online survey approximately one week after the virtual orientation. Families who completed the survey were contacted by a research team member and asked to participate in a followup interview via Zoom or phone approximately two weeks later. Details about the sample of participants, virtual orientation events and research instruments are described in detail below. Analyses were conducted with the sample with complete data (see Results on page 3). However, attrition analyses were conducted to compare survey responses in the partial data (survey only) vs complete data (survey and interview) samples (see page 4).

\section{Sample}

In collaboration with three partner PBS stations, a total of 60 families were recruited through stations' community partnerships to participate in the station study. The stations were located in various rural and urban settings across the country. As an incentive to participate, 
each family received a Kindle Fire tablet. Families also received a $\$ 25$ gift card at the end of the study.

In the complete data sample, the majority of parents/caregivers identified their race as White (65.52\%), followed by Black or African American (24.14\%). Of the remaining parents/caregivers, one identified as American Indian or Alaska Native and White and three preferred not to answer this item. None of the parents/caregivers reported being of Hispanic, Latino, or Spanish origin. All parents/caregivers indicated English as both their first language and the language most often spoken by children at home. Most parents/caregivers indicated having only one child between 4 and 7 years old (75.86\%), with most remaining parents/caregivers (17.24\%) indicating they had two children within this age group. Only two parents/caregivers reported having more than two children within this age group. The average age for children in the sample was 5 years old.

Parents/caregivers reported that their children regularly use technology and generally had positive views on using technology to support children's learning. Nearly all of them (96.42\%) indicated their children watch and/or play educational content often or every day. Most agreed or strongly agreed that technology provides young children with information that they might not get at home or school as well as helps teach young children skills they will need in the future. Although parents/caregivers reported their children used technology frequently, $89.28 \%$ reported that the Splash and Bubbles for Parents app was very different or somewhat different from other apps they have used.

\section{Virtual Orientation Events}

The content of these events was intentionally designed to address the findings from the field study that pointed to specific supports that may improve and deepen families' engagement with the app. To attend to this goal, researchers developed study materials to introduce the station leaders to the Splash and Bubbles resources as well as the research components of the study. The research team then worked closely with PBS station partners to walk through materials together and prepare for the virtual family events.

After planning and preparation with the research team, station leaders facilitated virtual events with those families who were recruited from their local communities. Before the virtual event, stations distributed Kindle Fire tablets to each family. These devices were intended to support parents as a second screen to watch the Splash and Bubbles show and/or access the Splash and Bubbles for Parents app. Stations also shared brief descriptions of the Splash and Bubbles resources to support parents in the preparation for the event.

Families were invited to join a Zoom meeting along with other study participants to learn about the app and their engagement in the research project. During the event, families were provided an orientation to the background and different features of the app (via PowerPoints that included screenshots and interactive images), along with support for how parents may engage in conversation and science learning together with their children. This included a step-by-step tutorial that highlighted each section of the app, how to access and use each component, as well as where to view the Splash and Bubbles show. Each event lasted approximately 30 minutes. Stations had different approaches for following up with families 
not in attendance. Station 1 conducted a separate virtual orientation with the two remaining families. Similarly, Station 3 held a virtual orientation with one family; for the other remaining families, the station leader shared the recording of the event with the families to view at their discretion.

\section{Data Collection Measures}

\section{Family Survey}

Each participating family was asked to complete an online survey approximately a week after the virtual orientation. This survey was designed to gather information about how families engaged with both the Splash and Bubbles show and the Splash and Bubbles for Parents app, including how they accessed and used the resources as well as their perceived affordances of the app for promoting science learning. This survey also intended to elicit families' attitudes, beliefs, and practices around the use of technology and media.

\section{Family Interview}

Families who completed the online survey were invited to join a 30-minute interview via Zoom or phone. The interview was designed to gather more detailed, qualitative data about how families used the Splash and Bubbles for Parents app. When technology platforms allowed, parents/caregivers engaged in a screen share to walk through their app use and provide typical navigation patterns. Interviews also elicited families' thoughts and feedback about the intended purpose of the app; affordance for supporting learning and conversations about science; and ideas for improvement or revision.

\section{Results}

Approximately two thirds (63\%) of the sample (38 families) completed the online survey. While the level of attrition in research with families tends to be high, the attrition rate was higher than expected, likely due to the COVID-19 health emergency. Some of the participating families shared they were experiencing health and/or stress-related issues that may have influenced their ability to participate in research activities. Of the 38 families who completed the survey, $76.32 \%$ (29 families) also completed the interview. This group of participating families is referred to as the complete data sample. Table 1 shows the distribution of families from each of the three stations by their participation in the study research activities. 
Table 1

Family Participation in Research Activities by Station

\begin{tabular}{|l|l|l|l|}
\hline & $\begin{array}{l}\text { Total Families in } \\
\text { Sample }\end{array}$ & $\begin{array}{l}\text { Number of Families with } \\
\text { Partial Data (survey only) }\end{array}$ & $\begin{array}{l}\text { Number of Families with } \\
\text { Complete Data (survey } \\
\text { and interview) }\end{array}$ \\
\hline Station 1 & 10 & 1 & 9 \\
\hline Station 2 & 15 & 6 & 9 \\
\hline Station 3 & 13 & 2 & 11 \\
\hline
\end{tabular}

As stated earlier, attrition analyses were conducted to examine whether there were any differences between the partial data group and the complete data group. Nine of the families who participated in the survey did not participate in the interview, again possibly due to the COVID-19 health emergency. Interestingly, a significant portion of these were Station 2 families (67\%). Of these nine families, seven indicated that they tried using the Splash and Bubbles for Parents app. In our analyses, we found that the partial data sample rated components of the app (e.g., Let's Talk About It conversation starters and Ocean Guide) slightly lower than their counterparts with complete data. In addition, slightly more than half of this sample $(57.14 \%)$ did not use the sync feature. Their reasons for not using sync were evenly split between choosing not to use it and trying to use it but encountering technical difficulties. Given these findings, it is important to note that it is possible that families with partial data found the app less valuable than families with complete data. Another consideration for attrition may be related to different levels of continued support for participating families following the orientation events. Station leads engaged in communication with families throughout the study to answer questions, as well as provide guidance and gentle reminders to complete study activities (e.g., app use, survey, and interview). Some stations provided more frequent support to families via email and phone communications, and engaged in more coordination with the research team.

\section{Splash and Bubbles show Engagement and Perceptions}

Parents/caregivers reported on their families' typical viewing practices with most engaged in some form of co-viewing: children either watched the Splash and Bubbles episodes with them/another adult (72.41\%) or with them/another adult and peers (17.24\%). Only a small percentage of parents/caregivers reported children watching on their own $(10.34 \%)$.

Although the sample is small, findings indicate that most families tend to jointly engage with 
media resources. This finding, coupled with the positive response to the show and the related learning opportunities, provides compelling evidence for the affordances of a second-screen app that can further support families' joint engagement with media and science discourse.

Overall, the Splash and Bubbles show was positively received by participating families. Most (89.66\%) reported that their child enjoyed the Splash and Bubbles show. All except one parent/caregiver either agreed or strongly agreed that their child learned about ocean science concepts while watching the show. In addition, most agreed or strongly agreed that their child wanted to do activities or learn more about the science ideas promoted in the show and asked questions while viewing. Only one parent/caregiver disagreed and one strongly disagreed that they engaged in science talk related to the Splash and Bubbles show with their child. A majority of families also agreed $(75.86 \%)$ or strongly agreed $(13.79 \%)$ that after watching the Splash and Bubbles show, they felt more confident engaging in science talk with their child.

\section{Splash and Bubbles for Parents App Use}

Almost all (96.55\%) of the participating families reported using the app in the survey. The one parent/caregiver that indicated not using the app in the survey subsequently reported using the app and some of its features during the interview (perhaps indicating they tried the app after the survey was complete). Families primarily accessed the app using a smartphone $(46.43 \%)$ or tablet $(42.86 \%)$ with the remaining percentage using both a smartphone and tablet.

In the survey, families were asked to indicate which section(s) of the app they used. The app includes three main sections: Dive In, Ocean Guide, and Activities.

\section{Dive In}

The Dive In section provides related content specific to each episode and includes the following components: Let's Talk About It (conversation starters to support science talk); We'll Learn About (science themes); Characters (information about the characters in the show and the creatures they represent); and Clips (short video segments from the show or a separate video clip related to a science theme referred to as an interstitial). 


\section{Ocean Guide}

The Ocean Guide contains additional science information from the show organized around the following components: Featured (specific environments and topics are highlighted); Characters; Environments (information about the habitats explored in the show); Topics (science concepts addressed in the show); and Clips.

\section{Activities}

In the Activities section, families can find short, engaging handson learning activities designed to extend children's understanding of the science ideas introduced in the show by connecting them to children's everyday experiences. Some activities include a "Snap It" component to encourage families to take photos (accessible through the Photo Journal) of their science adventures.

Most families used at least two of the app sections described above $(78.57 \%)$ while the remaining families used only one section (21.43\%). Of the six families that used only one section, two thirds reported using Dive In with the remaining third using Activities. Findings specific to each section are detailed later in the report.

\section{(2)}

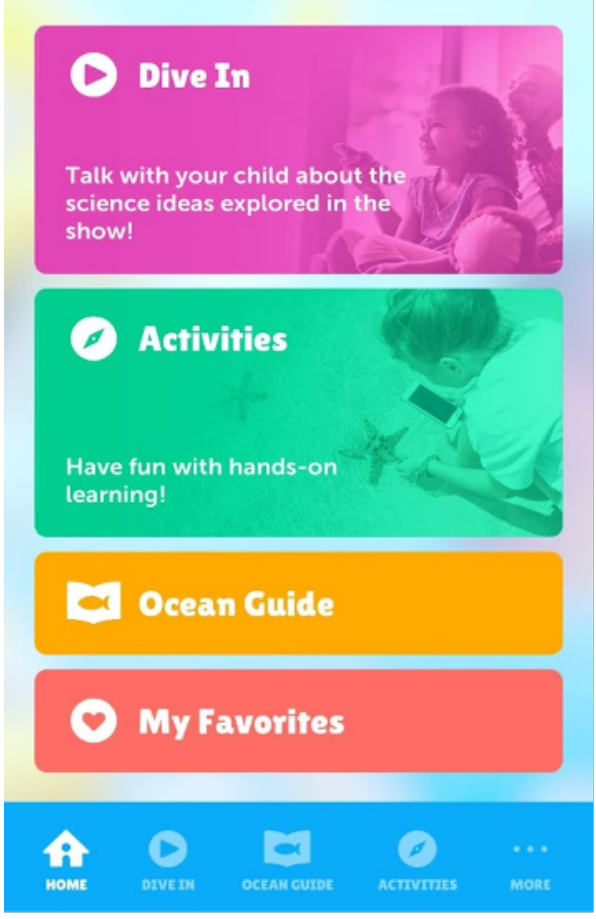

Sync

Families were also asked whether they used the sync feature while watching an episode. This unique feature of the app utilizes ACR technology to allow automatic syncing to the episode being watched, serving as one potential entry point for families to access related content easily and in real time. Although episode content can still be accessed without the use of the sync, its uptake may be indicative of a particular use case, namely families who are more inclined to engage with media while viewing the show together.

Approximately two thirds of the families (64.3\%) reported using the sync in the survey. At the time of the interview, fewer families $(44.83 \%)$ reported being able to use the sync feature, however. Of the 13 families that reported using the sync feature during the interview, most enjoyed the feature and found it very helpful for accessing related content while watching episodes.

- "I have not seen anything like it. Sync has not been in any other apps/shows-that is a total game changer. It was so cool to connect that way."

- "Like the sync button works really well so I don't have to sift through all the episodes to find the one we're watching."

Interview data helped uncover explanations for why slightly more than half of the families $(55.17 \%)$ did not use the sync feature. Of this group, less than half $(43.75 \%)$ were aware of the 
feature. More than one third of the families (37.5\%) that reported not using the sync feature described having technical difficulties or being unable to figure out how to use the feature. A couple of these families indicated that they did not use a second screen to access the app, but rather watched the show and used the app on the same device (which would preclude them from being able to use the sync feature). Finally, some families reported that they chose to manually look up the episodes on the app or did not explicitly mention whether or not they used the sync feature.

\section{Overarching Perceived Benefits of the App for Learning and Joint}

\section{Engagement}

Families generally had positive views and found the app helpful in supporting child learning and facilitating conversations with their child/children. A majority of them (86.2\%) identified the app's purpose as child learning, and of these, half also noted the app supported joint engagement. This was evident in parents/caregivers' responses in the interviews. For example, one parent/caregiver explained the app is intended to support them in having conversations with their children that are helpful to their learning.

In the survey, families were asked to rate on a scale from 1 (not very helpful) to 5 (very helpful), how much they think the Splash and Bubbles for Parents app supports parents to help children learn about science. All participants rated the app either a $4(46.4 \%)$ or a 5 (53.6\%). Most families also agreed (64.3\%) or strongly agreed (25\%) that after using the Splash and Bubbles for Parents app, they felt more confident engaging in science talk with their child.

\section{Figure 1}

Percentages of Agreement and Disagreement with Parents/Caregivers Feeling More

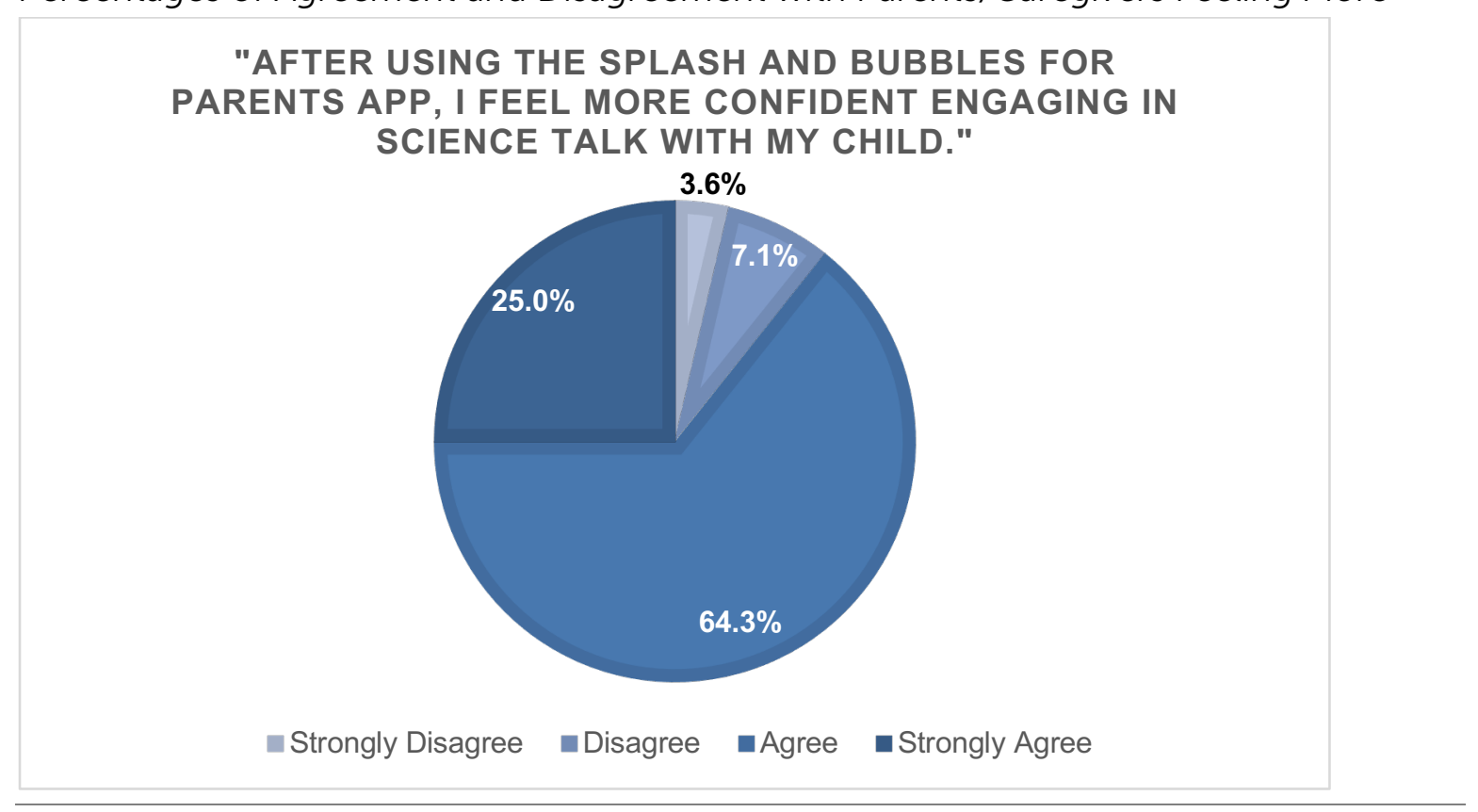


Interview data was analyzed to further understand the survey results and gain insight into families' thoughts about and use of the app. Almost all (96.55\%) families thought the app helped them and their child/children learn science and have rich conversations. Several families mentioned in their interview that the conversation starters in the app gave them ideas on what to talk about with their children, and they enjoyed being able to learn with their children.

- "Had good conversations with 6-year-old teaching me about what he was seeing. He's a really good reader and wanted to read it [the app] himself and talked about it for days after the first time. Would ask if I remembered certain characters and things about them."

Although a couple of families indicated in their survey that they did not feel more confident engaging in science talk with their child after using the app, they still found the app helpful and engaged in conversations to an extent. One parent/caregiver explained that the app helped them start conversations, but maintaining them was challenging given they found the language used in the show and the app was too advanced for their children. Another parent/caregiver indicated age being a limiting factor because their 4-year-old child was less engaged than their 7-year-old child. An example illustrating how conversations emerged for this particular family is shared in the Ocean Guide section below.

\section{Dive In}

At the time of the survey, nearly all families (92.86\%) indicated using the Dive In section of the app. The two families that did not report using the Dive In section when they completed the survey subsequently reported using the Let's Talk About It conversation starters in the Dive In section during their interview. One of these families spoke about the importance of conversations for supporting children's learning, stating the app "made her job so much easier" by facilitating conversations and helping her child learn. Another family thought the conversation starters helped get the conversation going and liked the definitions in the app. This family also reported not thinking to ask questions during the episode because she did not want to interrupt, but plans to use them during episode prompts in the future even if she has to pause it to talk to her child about it.

Of the 26 families that reported using the Dive In section, most indicated the Let's Talk About It section (73.08\%) as the most helpful section followed by Characters (15.38\%), Clips (7.69\%), and We'll Learn About It (3.85\%). When asked to rate on a scale from 1 to 5 (5 means very helpful) how much they think the Let's Talk About It conversation starters support parents in helping their children learn science, most rated the prompts a $4(57.7 \%)$ or $5(26.9 \%)$. A smaller percentage rated the prompts a 3 (15.4\%).

- "Liked that I could look at the app and know what's going on without having to watch a children's program and still connect with her with less effort. Nice cheat sheet."

- "What I liked about the app was basically how it gives you questions and everything that you'll be able to provide your kid or what to expect when watching the video." 
During the interviews, families were asked to share their experiences using the conversation starters and provide feedback on the different sets of questions (during episode, after episode, and repeat viewing) in the section. In alignment with the survey results, families that reported using the Let's Talk About It conversation starters generally found them helpful and engaging. Further analyses also suggested variability in preferences and use cases for the different prompts. For example, one family mentioned that they preferred the during episode questions because they were able to use those with their younger (3-year-old) child; they allowed them to check if they were paying attention and understanding the basic points in the episode. Whereas other families noted that they preferred the after episode prompts because they felt asking questions during the episodes was interrupting their children while they were watching the show. At least one family talked about liking both the during and after prompts and found them helpful in different ways. More specifically, this parent/caregiver said that during episode questions were fun for both of her kids (6- and 9year-olds) because it was like a scavenger hunt, while the after episode questions were able to get her 6-year-old in thinking through what he had seen and learned from the episode.

Approximately half $(53.8 \%)$ of the families indicated that they were either not aware of the repeat viewing questions, which suggests additional support around how to access them may be useful, or they did not watch the episodes more than once to use them. Of the five families that used the repeat viewing questions, two indicated that they liked the questions because they were more specific and went in-depth about the concepts from the show.

- "Second viewing-more depth about things. Son noticed different things every time, so questions brought out more things he noticed."

\section{Ocean Guide}

More than half of the families (57.14\%) reported using the Ocean Guide section in the survey. Families endorsed different sections of the Ocean Guide as most helpful, indicating widespread user/learner variability. Of the 16 families who reported using the Ocean Guide in the survey, the largest proportion (62.50\%) were evenly split, selecting either Characters or Environments as most helpful, followed by Topics (18.75\%), Clips (12.5\%), and Featured (6.25\%). The interviews provided important context for understanding why the Ocean Guide section resonated with families. For instance, the one family who was aware of the Dive In section (where families can access the conversation starters) expressed not using the conversation starters because they found them more suitable for older children. In the interview, she shared that her 3-year-old liked all the character images and could recognize them, thus substantiating why their survey response indicated Characters as the most helpful Ocean Guide section.

As evidenced in the interviews, families were able to utilize the Ocean Guide components in ways that were still helpful in promoting science learning and conversation with their children. The Ocean Guide sections presented additional opportunities for families to engage in science talk together. For instance, one family liked the Characters and Environments, and specifically noted the Environments allowed them to see places they do not typically visit. This family also appreciated being able to access information and inspire questions about 
specific animals/characters. Another parent/caregiver who liked Environments shared in their interview that they talked about the different habitats, and that her child knew a lot about giant kelp. Finally, a family noted that different components of the Ocean Guide were helpful to children of different ages; in this instance, the older child (7 years old) had more interest in the scientific ideas in Topics while the younger child (4 years old) preferred to talk about the creatures in Characters.

\section{Activities}

Approximately two thirds of the families (65.52\%) indicated using the Activities section on their survey. Of these 19 families, a majority (78.95\%) reported the activities as the most helpful section followed by the Photo Journal (21.05\%). Over two thirds (68.42\%) indicated completing two or more activities with the remaining (31.58\%) completing one activity. By the time of the interview, a slightly higher percentage of families $(75.86 \%)$ mentioned using the Activities section including four who had not used it at the time of the survey.

Furthermore, the survey and interview findings demonstrate families' generally positive perception of the activities, namely their overall enjoyment and helpfulness. Families were asked to rate how much they think the activities support parents to help children learn about science on a scale of 1 to 5 (5 means very helpful) on the survey. The average rating was 4.3 with no families rating lower than 3.

From the interviews, we learned more about how families engaged with the Activities section. Similar to the Ocean Guide, families were interested in the Activities section for a variety of reasons, thus utilizing it to meet their specific needs.

- "Love that it brings in the environment and conservation. It is so important to know about how to protect the environment and children from all places can connect with that. Also, like that activities suggest different materials around the house. The activities can have differentiation across age ranges."

The activities, along with the Photo Journal, were another avenue for families to engage in scientific discourse. One family who selected the Photo Journal as the most helpful section expressed really appreciating the activities, specifically referencing that the Photo Journal "allowed for opportunity to incorporate things she sees every day." This not only made the content relatable for the child, but also demonstrated to the parent/caregiver that the child was grasping the concepts. Other families who engaged in the activities were still able to have conversations even if they were unable to find animals to document in the Photo Journal or were unsure as to whether the activities related to certain episodes. 